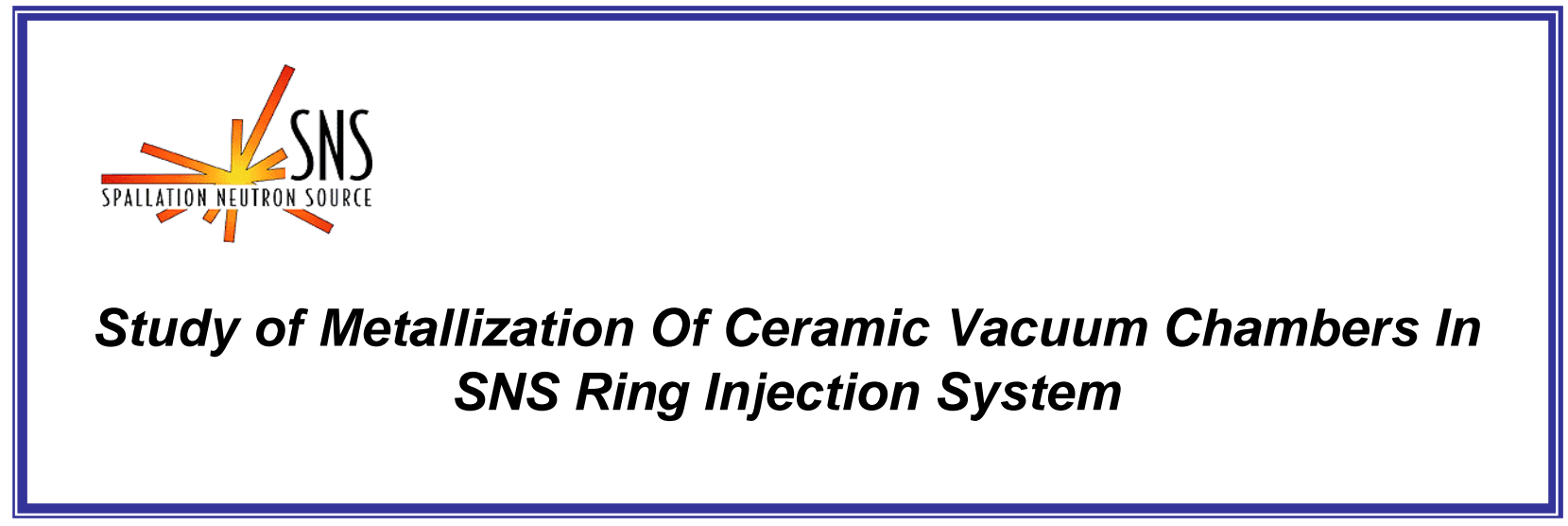

BNL/SNS TECHNICAL NOTE

NO. 99

P. He, H.C. Hseuh, R. Todd

BNL, Upton, NY 11973, USA

August 30, 2001

COLLIDER-ACCELERATOR DEPARTMENT BROOKHAVEN NATIONAL LABORATORY UPTON, NEW YORK 11973 


\title{
STUDY OF METALLIZATION OF CERAMIC VACUUM CHAMBERS IN SNS RING INJECTION SYSTEM*\#
}

\author{
P. He, H.C. Hseuh, R. Todd \\ Collider-Accelerator Department, BNL, Upton, NY 11973, USA
}

\begin{abstract}
The pulsed kicker magnets used for the injection of $\mathrm{H}^{+}$ into the SNS accumulator ring consist of ceramic chambers to avoid harmful inductance generated by the rapid magnetic field rise time. The inner surfaces of these ceramic chambers will be coated with a thin metallic layer ( $\mathrm{Ti}$ or $\mathrm{TiN}$ ) to meet the requirements of both the penetration of pulse magnetic field and small beam coupling impedance, also reduce the secondary electron yield. This paper describes the metallization facility for the $0.85 \mathrm{~m}$ long ceramic chamber which has been developed at BNL. The facility produces thinknesses up to 10 microns titanium or titanium-nitride by means of DC magnetron sputtering.
\end{abstract}

\section{INTRODUCTION}

Brookhaven is undertaking the design and construction of the SNS accumulator ring. Ceramic chambers and ferrite kicker magnets were developed in SNS njection system. Ceramic chambers are used to allow external time varying fields to penetrate the vacuum chamber, while a thin metallic coating is required to carry the beam induced image currents thus protecting external components. The coating material and thickness are chosen based on the following requirements[1]:

(i) external fields must penetrate the coating

(ii) the risetime of the external field must be preserved

(iii) electrical continuity to the adjoining vacuum chambers must be provided

(iv) the beam fields must be contained

(v) the film must tolerate magnet pulsing (remain adherent, no arcing, etc)

(vi) subject to acceptable heat load: ohmic heating from the beam image current

(vii) acceptable heat load: eddy current heating from magnetic pulsing

(viii) also acceptable heating from absorption of HOM power generated by discontinuities at the joints between the chamber and the surrounding vacuum system. low secondary electron yield(SEY).

The ceramic chambers, totaling six for the injection kicker system, have a $16 \mathrm{~cm}$ (ID) and total length and are $0.83 \mathrm{~m}$ long. Alumina ceramic was chosen as vacuum chamber because of its greater mechanical strength, relative high thermal conductivity, and best braze metallization. The metallization must have a minimum resistivity to avoid strong attenuation and deformation of the magnetic field. A very homogenous and well adherent layer is required otherwise the high voltage pulse induced by the pulsed magnetic field would cause destructive sparks along the coating. Titanium, Titanium-Nitride, or a combination of both, have been chosen as the coating material for its high melting point and it's reactivity, resulting in good adhesion from chemical bounds to the silicon phase of the alumina, assuring good adhesion. Later, ORNL modify SNS injection kicker œramic chamber coating specification and recommend to coat copper $\sim 750 \mathrm{~nm}$ firstly for getting a flange-to-flange DC resistance of $0.04 \mathrm{Ohms}$, in addition the surface should be coated with $100 \mathrm{~nm}$ TiN, as required for all other SNS ring vacuum chambers, for the reduction of the secondary electron emission.

This paper presents magnetron sputtering system and some preliminary test results.

\section{SPUTTER COATING DEVELOPMENT}

(a) Coating method and magnetron cathode

Original coating method was selected as the conventional diode sputtering, because it is much simple and easy to set-up, just need to introduce a cathode over the total length into the chamber and apply a DC or RF current at several kilovolts. The disadvantage is that because of the narrow aperture of the chamber the process has to take place at a relatively high pressure in order to be able to overcome the cathode dark space. However, the higher the gas pressure, the lower the deposition rate. This results in more impurities accumulating in the coating due to increased site competition with contaminants. Sputtering with a magnetron cathode can resolve these problems. By using a magnetic field which crosses the electric field perpendicular, the plasma density in the discharge region can be raised by more than two decades over the level obtainable with simple diode discharge, therefore a large amount of power can be supplied at a lower target voltage. Because of the high plasma density, an important restriction of the cathode sheath thickness is lifted, in consequence much less space is needed between the cathode and the substrate for maintaining the discharge. In a magnetron discharge more 
than $90 \%$ of the total energy input is dissipated on the target and is eliminated by the cooling water. The remaining heat, produced by the collected electron current, the heat of condensation of the metallic vapour and the bombardment by energetic neutrals from the plasma is distributed between the anodes and along the section of the chamber, which is progressively metallized within a couple of hours.

The magnetron diode is a sputter device where magnets, installed behind the target's surface create a magnetic field. The field lines originate and terminate at the cathode, and in between the two magnetic poles there is a region where they are parallel to the surface. As in an ordinary diode discharge, electrons are accelerated in the cathode's electric field and ionise gas atoms present. Positive ions then strike the cathode surface and liberate new electrons by the mechanism of secondary emission. An ionization avalanche takes place and subsequently a steady electric discharge is between the cathode and the anode. Electrons follow a helical motion along B field lines, also electrons are reflected back and forth between points where the lines enter and leave the cathode surface. The E x B drift causes the electrons to move parallel to the cathode surface in a direction determined by the magnetic field. Finally, electrons escape from the magnetic trap and flow to the anode after having produced a number of elastic and inelastic collisions with gas atoms. Positive ions strike the cathode also sputtering metallic atoms from it. These atoms are ejected with substantially higher kinetic energy than thermally evaporated ones and from well adherent deposits on any solid surface installed in the proximity. This is a final scope of the operation.

The cathode can take various forms: "planar" or "cylindrical" and "hollow" cathodes. Much work has been done on the formation of $\mathrm{Ti} / \mathrm{TiN}$ by magnetron sputtering as an industrial hard coating using planar electrodes and magnets. However, due to our chamber geometry, a novel approach had to be taken in developing a cathode target with suitable magnetic field characteristics. Commercially available Alnico magnets $1 \frac{1 / 4}{4}$ " diameter and 2 " in length were inserted in a 11/2" diameter, grade-2 titanium tube. A $1 / 2$ " hole in the center of the magnets allowed for water cooling of the cathode. The magnets were stacked with opposing poles using non-ferrous $1 / 2$ " spacers resulted in a looping magnetic field of sufficient strength to project from the cathode surface. This "low cost" cathode (Fig.1)

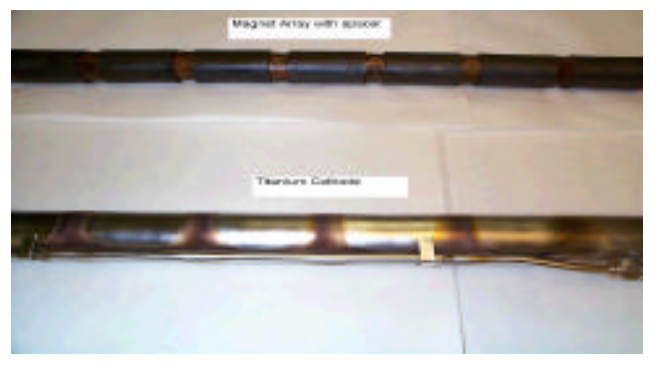

Fig.1. Magnetron and Titanium target worked in conjunction with a $10 \mathrm{KW} \mathrm{DC} \mathrm{power} \mathrm{supply} \mathrm{to}$ produce the satisfactory field and discharge plasma.

(b) Sputtering facility and vacuum system

The chamber used to develop the SNS Half-Cell chamber TiN coating was used to coat the ceramic pipes [2]. The diagram of coating system was shown in Fig.2.

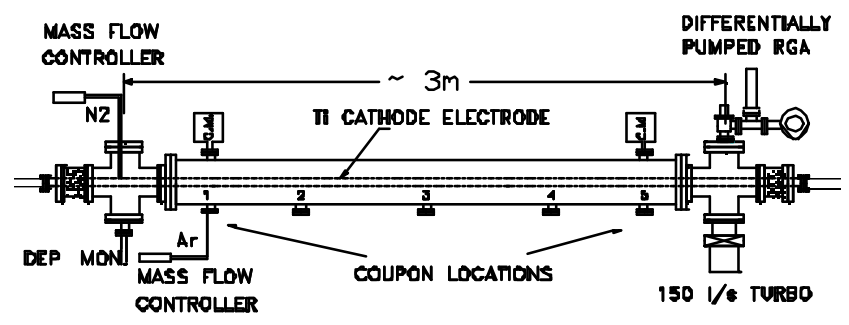

Figure 2. Setup of Ti/TiN coating of test chamber

Pumping is achieved by a 1501/s turbomolecular pumping station. The gas inlet system was installed at the left side, equipped with mass flow controllers for both argon and nitrogen. The viewing ports allow observation of the plasma. A cold cathode gauge is used to monitor the vacuum pressure prior to metallization. A high pressure residual gas analyzer capable of operating and discharge pressure, was used to check the residual gas composition before the coating and to monitor nitrogen consumption during the coating.

\section{(c) Sputtering procedure}

We did two test runs using the above (Fig.2) sputtering facility. First run we put one ceramic tube inside test chamber and second run we put two glass tube so as to comparison, one with Al stripes and another one without Al stripes.

After the ceramic or glass tube mounting and aligning into the sputtering installation, the chamber is evacuated and baked by mean of external heating tapes to $250{ }^{\circ} \mathrm{C}$ about 48 hours to remove the water vapour and surface contaminants, and then start coating, during the sputtering, this temperature is maintained.

Following the residual gas analysis, just make sure no any organic contamination, the Ar (for Titanium coating) or Ar and $\mathrm{N}_{2}$ (for Titanium Nitride coating) was injected into the test chamber, the flow rate was adjusted by the mass flow controller. Gas purity is better than $99.9999 \%$ for both gases. In the first test run(TiN coating on ceramic tube), the flow rate is $13.5 \mathrm{sccm}$ for Ar and $7.5 \mathrm{sccm}$ for $\mathrm{N}_{2}$ respectively; in the second run( $\mathrm{Ti}$ coating on glass tube), we only injected Ar at $13.5 \mathrm{sccm}$.

The plasma is then ignited by slowly adjusting the DC power supply to $1 \sim 3 \mathrm{kw}$ output power. Typical voltage and current setting for TiN coating are $340 \mathrm{~V}, 10 \mathrm{~A}$ and for Ti coating are $290 \mathrm{~V}, 5 \mathrm{~A}$. The deposition rate are about $2.8 \mathrm{~A} / \sec (\mathrm{Ti})$ and $0.7 \mathrm{~A} / \mathrm{sec}(\mathrm{TiN})$, measured by thim film monitor. In the first test run, we spent 24 hours to get 
about $6 \mu \mathrm{m}$ TiN coating on ceramic tube, in the second run, we get $6 \mu \mathrm{m}$ Ti coating on glass tube in 6 hours.

\section{PRELIMINARY TESTS}

Throughout the development of the coating procedure, one ceramic tube and two glass tube were coated for various sputtering conditions, the properties of Ti/TiN films were tested.

\section{(a) Adhesion test}

The coating adhesion to the surface of the substrate is one of the most important properties. Two methods have been used for adhesion test. The first one is the simple "scotch-tape test" in which adhesive tape is attached to the film and pulled quickly in an attempt to remove the film. The second more demanding test requires scribing a grid of finely spaced cuts through the film down to the substrate(typically a $4 \times 4$ grid with cuts spaced $\sim 1 \mathrm{~mm}$ apart), and then this portion of the film eas subjected to the tape test. Generally, if a film passed this test, it could not be removed by any others means. Both of the methods have applied to test the ceramic tube and glass tube. The results show the good adhesion to the ceramic tube TiN coating, but poor adhesion to the glass tube Ti coating. Probable due to the different surface condition of two kinds of tube. But it is ok, we don't really care about the Ti coating film adhesion on the glass tube, the purpose of using glass tube as test tube, because its cheaper cost and its transparency so as to we can watch the plasma status during the sputtering by means of the viewing ports.

\section{(b) Resistivity measurement}

The resistivity of the metal layer is measured by a four-point probe, consisting of four pins in line, connected to a bridge Ohm meter(Fig. 3)

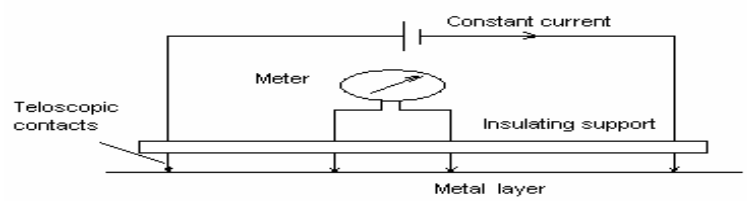

Fig.3. Resistivity measurement by four-point probe

For the ceramic tube, using the four-point probe, we measured the resistivity of TiN coating at the end of ceramic tube, get the value is $45 \mu \Omega$.cm, let's assume the resistivity is constant, moving the the four-point probe along the $\mathrm{z}$ direction, we get the thickness distribution(Fig.4). From Fig.4, we see the thickness uniformity is really bad, the various factors which affect thickness uniformity were discovered in the development stage, such as magnetic field strength, pumping speed and cathode uniformity, etc, but main factor here is due to the electric field built up inside the ceramic tube, very weak plasma discharge produced in the middle of the tube.

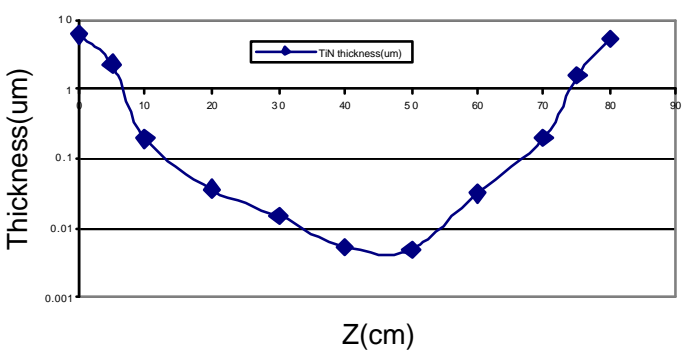

Fig.4. Ceramic tube TiN coating thickness distribution

It was necessary to determine if the coating would have acceptable thickness uniformity along the length. In the second test run, we put two 1" width Al stripes attached to the inner wall of glass tube and grounding them, it works as anode screen. We choice the glass tube because we want to see the plasma discharge during the coating. Two glass tube were installed inside the test chamber, one with Al stripes(\#2 glass tube) and another one without Al stripe(\#1 glass tube). During the coating, we clearly see no plasma discharge in the middle of \#1 tube, but very stable discharge in the \#2 tube. Fig. 5 shown the two glass tubes after coating.

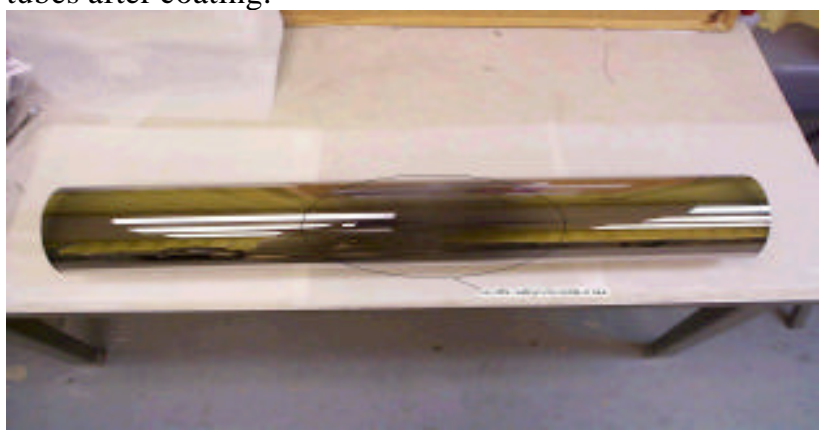

Fig. 5a. \#1 glass tube without grounding Al stripe

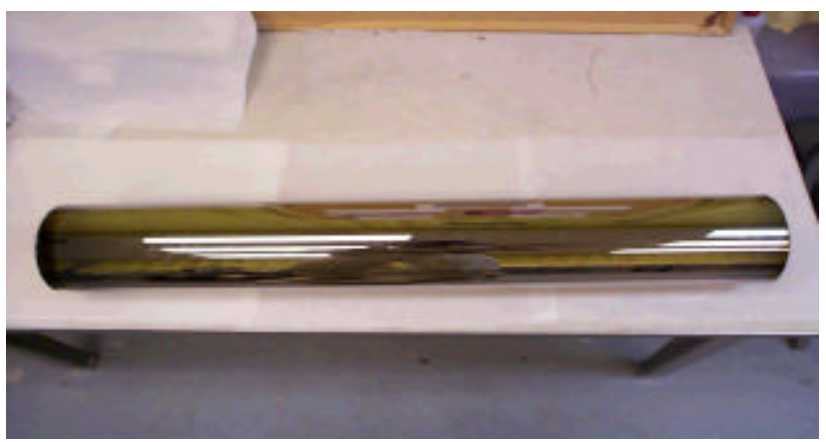

Fig. 5b. \#2 glass tube with grounding Al stripes

Using the four-point probe measure the resistivity and the thickness uniformity. The $\mathrm{Ti}$ thin film resistivity is 
about $95 \mu \Omega$.cm and the thickness distribution was shown in Fig. 6. The longitudinal resistance is $1.59 \Omega / 141 \Omega$ w/o Al stripes.

Thickness Distribution

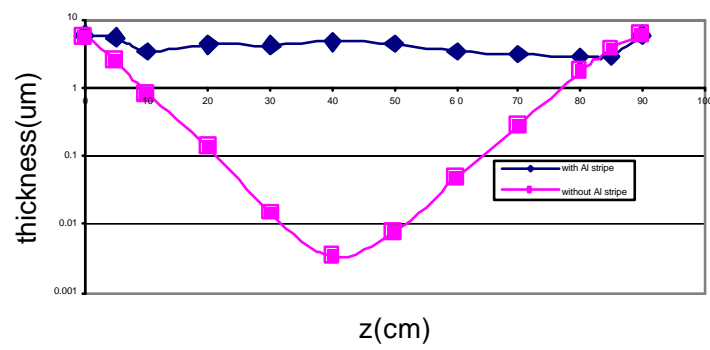

Fig.6. Thickness distribution of two glass tube

As shown in Fig.6, \#1 tube without Al grounding stripes, the thickness uniformity is very similar with ceramic tube, but \#2 tube with Al stripes get much better longitudinal thickness uniformity, so we knew the main factor which influenced the thickness uniformity is the electric field built up inside the glass tube.

\section{FUTURE PLAN}

Next test run, we'll use the special screens in the left and right side of the chamber, this non-uniform coating reduce the eddy current losses comparing to the uniform coating, the shield pattern is under developing.

After the ceramic tube metallization, we also need high voltage test, the thin metal layer should be pulsed for several million pulses without failure. A short full aperture kicker magnet model, producing the nominal pulsed magnetic field, will be used as the test bench. The performance of this test should fully representing kicker operational conditions including pulsed power supply.

\section{SUMMARY}

DC magnetron sputtering has been developed to coat the SNS ring injection kicker ceramic vacuum chamber with $\mathrm{Ti} / \mathrm{TiN}$ or $\mathrm{Cu} / \mathrm{TiN}$. The difficulties in producing a film with uniform properties within the long ceramic chamber have been overcome with the development of a unique special screen, also the low resistivity thin film coating has been get.

\section{ACKNOWLEDGEMENT}

The authors would like to thank C-A Vacuum Group in setting up the experiment.

\section{REFERENCES}

[1] Alex. Chao, M. Tigner, Handbook of Accelerator Physics and Engineering
[2] P.He, H.C. Hseuh, R. Todd, et. al., Proc. of 2001 Particle Accel. Conf., Chicago, USA(2001).

[3] M.Mayer, H.Kuhn, G.H.Schroder, et. al., European Particle Accelerator Conference, Rome, Italy(1988)

[4] T.Mimashi, N.Iida, M.Kikuchi, H.Nakayama, et. al., European Particle Accelerator Conference, Vienna, Austria(2000) 